\title{
New horizons for the synthesis of nanoparticles: Germanium nanoparticles from metastable GeBr-solutions
}

https://doi.org/10.1515/mgmc-2021-0026

received June 18, 2021; accepted August 06, 2021

\begin{abstract}
We present the synthesis and characterization of halide-terminated colloidal Ge nanoparticles of 2-10 $\mathrm{nm}$ with a narrow size distribution, synthesized via a novel reaction route. The nanoparticles are prepared by the disproportionation reaction of metastable $\mathrm{Ge}(\mathrm{I}) \mathrm{X}$ solutions and are obtained in a maximum yield of $79 \%$. Control of the nanoparticle size is achieved by varying the aging time and/or temperature. The halide termination of the nanoparticles is a perfect prerequisite for further surface functionalization but also leads to a high sensitivity of the germanium nanoparticles to water and air.
\end{abstract}

Keywords: germanium, nanoparticles, size-control, metastable subhalide

\section{Introduction}

Different chemical methods have been introduced for the synthesis of a variety of nanomaterials, whereby the control of the physical properties of the synthesized nanomaterial is a key issue for actual applications (Goesmann and Feldmann, 2010). The properties of nanoparticles and of nanomaterials have agitated scientists and engineers due to their huge potential

\footnotetext{
* Corresponding author: Andreas Schnepf, Institute of Inorganic Chemistry, University of Tuebingen, Auf der Morgenstelle 18, D-72076 Tuebingen, Germany, e-mail: andreas.schnepf@uni-tuebingen.de Viktoriia Slynchuk and Dominik Naglav-Hansen: Institute of Inorganic Chemistry, University of Tuebingen, Auf der Morgenstelle 18, D-72076 Tuebingen, Germany

Martin Hodas and Frank Schreiber: Institute of Applied Physics, University of Tuebingen, Auf der Morgenstelle 10, D-72076

Tuebingen, Germany
}

applications in different fields (Campelo et al., 2009; Freund et al., 2018; Grzelczak et al., 2019; Lin, 2015; Loza et al., 2020; Stratakis and Garcia, 2012). Recently, a review about the transfer of knowledge to industrial applications was published by Modena et al. (2019). Within the field of nanoparticles, semiconducting ones have received considerableattention (Scheele etal., 2015; Weller, 1993) and for the last two decades, germanium nanoparticles have been of interest for a wide range of scientific initiatives. Colloidal Ge-nanoparticles are thus an attractive class of nano-sized building blocks from which it is possible to create complex materials with unique properties for a variety of applications in areas that include optoelectronic, (Ruddy et al., 2010; Talapin et al., 2010) energy conversion ( $\mathrm{Wu}$ et al., 2016), and biological applications (Bhattacharjee et al., 2013; Fan and Chu, 2010). For example, germanium nanoparticles can be used as non-toxic luminescent nanoparticles (Carolan and Doyle, 2014; Shirahata et al., 2013). An important aspect during the synthesis and application of semiconductor nanoparticles is the stability of the colloidal suspension (McVey et al., 2017; Rogach et al., 2007).

Germanium nanoparticles are difficult to synthesize due to the high crystallization temperature of the material and strong covalent bonding between the atoms. In early reports about colloidal germanium nanoparticles, strong reducing agents like $\mathrm{LiAlH}_{4}$, or $\mathrm{NaBH}_{4}$ were typically used under harsh reaction conditions (reaction temperatures up to $300^{\circ} \mathrm{C}$ ) to react with $\mathrm{Ge}(\mathrm{II})$ and $\mathrm{Ge}(\mathrm{IV})$ species like germanium oxide or germanium halide salts, such as $\mathrm{GeX}_{2}$ or $\mathrm{GeX}_{4}(\mathrm{X}=\mathrm{Cl}, \mathrm{Br}, \mathrm{I})$. The synthesis via the solid-state disproportionation of $\mathrm{Ge}(\mathrm{II})$-precursors like a $\mathrm{GeO}_{\mathrm{x}}$ glass was reported by Panthani (Wang et al., 2019). Kauzlarich described the synthesis and characterization of $\mathrm{Ge}$ nanoparticles in a mixed solvent system, which affects the size and crystallinity of the nanoparticles formed (Bernard et al., 2018). Some methods are based on microwave- 
assisted reduction in solution (Smock et al., 2020). Boyle have studied methods which are based on a thermal disproportionation of $\mathrm{Ge}(\mathrm{II})$ precursors at $300^{\circ} \mathrm{C}$ (Gerung et al., 2005; Lambert et al., 2007). The surfactant-assisted solution reduction was reported by Chiu and Huang (2009). In a recent publication Delpech and Madec describe the synthesis and characterization of symmetric and nonsymmetric bis-amidinato-germylene $\mathrm{Fe}(\mathrm{CO})_{3}$ complexes, and the symmetric bis-germylene $\mathrm{Fe}(\mathrm{CO})_{3}$ complex, which have been applied successfully in the synthesis of iron germanide $\left(\mathrm{Fe}_{2} \mathrm{Ge}_{2}\right)$ nanoparticles in a crystalline core/ amorphous shell structure (Sodreau et al., 2019).

A major challenge for colloidal nanoparticles remains the ability to control shape, size and uniformity. In the following, we describe a completely new synthetic procedure to synthesize well defined and soluble germanium nanoparticles (GeNP). The GeNP are thereby synthesized by the disproportionation reaction of metastable $\mathrm{Ge}(\mathrm{I}) \mathrm{Br}$ solutions, which are available via a preparative co-condensation technique (Schnepf and Köppe, 2002; Schnepf, 2005). These nanoparticles are readily dispersed and colloidally stable in tetrahydrofuran (THF). The control of the nanoparticle size is studied and adjusted by variation of the reaction parameters temperature and time.

(a)
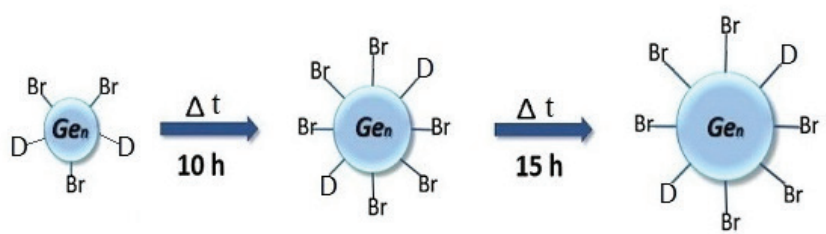

(b)

Temperature $\left({ }^{\circ} \mathrm{C}\right)$

- $35=45=55$

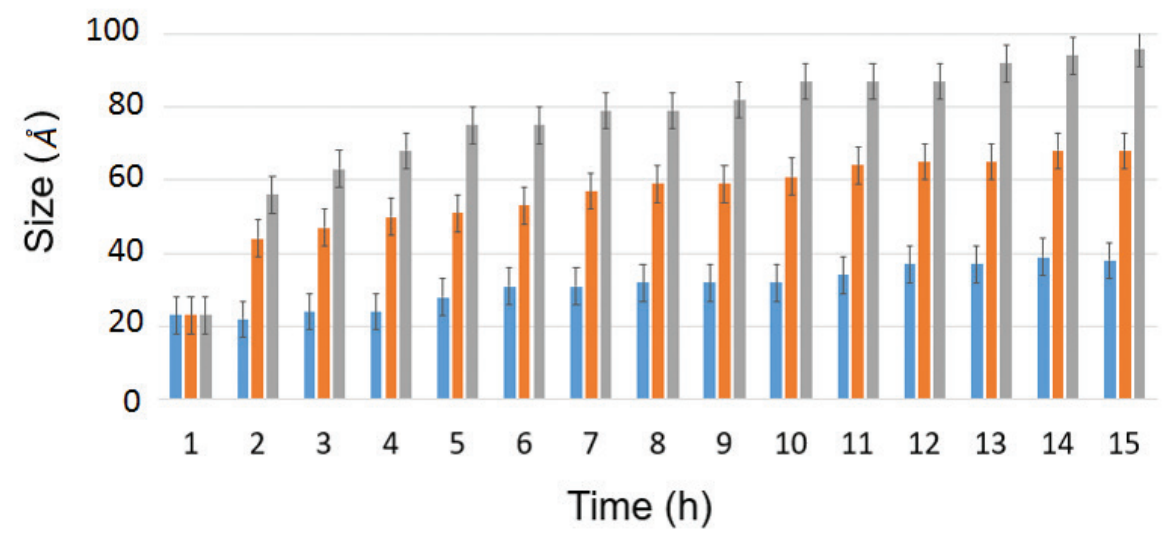

Figure 1: (a) Scheme of the GeNP size development $\left(D=\right.$ donor $\left(P^{n} B u_{3}\right)$ ). (b) Time dependence of the size evolution of the GeNP during the disproportionation reaction at three different temperatures of the reaction solution $\left(35^{\circ} \mathrm{C}, 45^{\circ} \mathrm{C}\right.$, and $\left.55^{\circ} \mathrm{C}\right)$, measured by dynamic light scattering (DLS). 

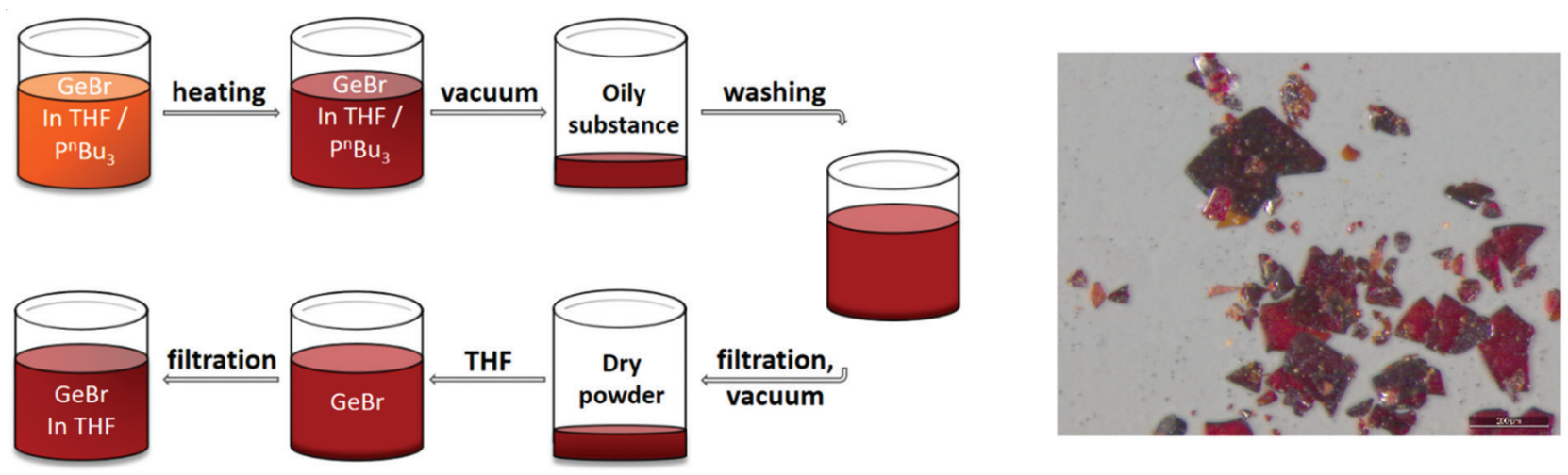

Figure 2: Schematic of the synthesis of colloidal GeNP including an image of dry powder.

and dispersed in THF as shown in Figure 2 and the experimental section. The size of the nanoparticles can be adjusted by varying the temperature and heating time for the altering of the metastable $\mathrm{Ge}(\mathrm{I}) \mathrm{Br}$ solution, resulting in deep red solutions (Figure S3b) of well-defined GeNP with a narrow size distribution. The hydrodynamic diameter of the particles is thereby measured by dynamic light scattering (DLS).

The main temperature limitation of this synthetic procedure is the boiling point of the solvent. Hence, toluene was tested as the solvent as well due to its high boiling point, allowing a broader temperature range for the synthesis of GeNP. However, the GeNP started to precipitate at $79^{\circ} \mathrm{C}$ which might be due to a bad solubility within toluene. The maximum hydrodynamic diameter of the nanoparticles before precipitating was thereby around $70 \AA$. When THF was used instead, the resulting GeNP with hydrodynamic diameters of up to 100 A give stable colloidal solutions for months. The so obtained germanium nanoparticles are afterwards isolated via the procedure outlined in Figure 2. The solutions and the dried GeNP are still very sensitive against moisture and air and must be handled under inert conditions, which might be due to accessible $\mathrm{Ge}-\mathrm{Br}$ units at the surface (Figure 1a). Nevertheless, under an inert atmosphere the nanoparticles are long-term stable in pure form as well as in solution.

The overall composition of the GeNP was determined by energy-dispersive X-ray spectroscopy (EDX) (Tables S1 and S2 in Supplementary material) and elemental analysis (Table S3), leading to an overall formula of $\left[\left(\mathrm{PnBu}_{3}\right)_{0.3} \mathrm{Ge}_{1.8} \mathrm{Br}\right]_{\mathrm{n}}$. During the work-up procedure outlined in Figure 2, a small amount of non-dispersible nanoparticles was observed, having the same composition as the dispersible ones. Hence, from the elemental analysis it is obvious that beside bromide substituents some part of the $\mathrm{PnBu}_{3}$ donor is left on the surface of the nanoparticles and continues to act as a surfactant, as also schematically shown in Figure 1.

After evaporation of the solvents, powder X-ray diffraction (PXRD) was used to verify the crystallinity of the nanoparticles, indicating that no crystalline core of, for example, $\alpha$-germanium is present (Figure 3). However, the width of the strongest peak (marked with a star in Figure 3) in the powder pattern indicates particle-particle correlations. In terms of sensitivity of the nanoparticles, the surface layer is partially oxidized to form mixed germanium-halide-oxide derivatives, resulting in increased intensity of impurity peaks (Johnson and Weller, 1999). Hence, after a prolonged measurement time, small peaks corresponding to the $\mathrm{GeO}_{2}$ diffraction pattern are observed (Uno et al., 1988) underlining the sensitivity of the particles to moisture and air as the $\mathrm{GeO}_{2}$ may result from a reaction of the particles with a small amount of water or oxygen during the measurement.

Small-angle X-ray (SAXS) scattering was used to determine the inter-particle distances and structure of the nanoparticles. Wide-angle X-ray (WAXS) scattering was used to determine the intra-particle structure. The capillary with solvent was measured first to obtain scattering pattern of the media without the GeNP. The measured pattern is presented in Figure 4a and shows no scattering intensity close to the direct beam. On the other hand, a strong signal in the WAXS regime is observed, corresponding to scattering of the quartz capillary. The measured reciprocal map of GeNP dissolved in THF and stored in quartz capillary is presented in Figure $4 \mathrm{~b}$. Compared to the SAXS pattern of the solvent in the quartz capillary alone, presented in Figure 4a, the scattering signal close to $\mathrm{q}=0$ comes from the GeNP. Hence the GeNP exhibit a typical disordered internal structure in solution, whereby Figure 4c corresponds to GeNP in solution with longer acquisition time to obtain better statistics from the scattering. Guinier analysis was applied to the measured reciprocal maps 
(a)

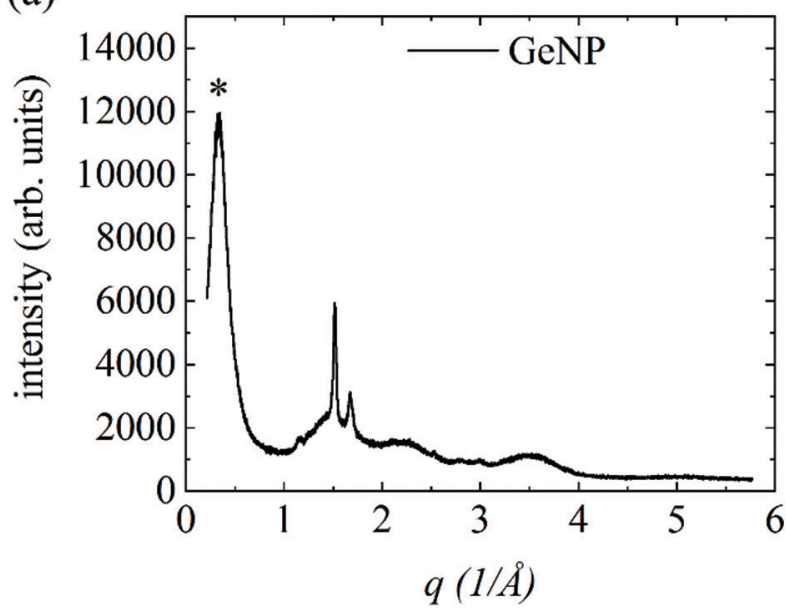

(b)

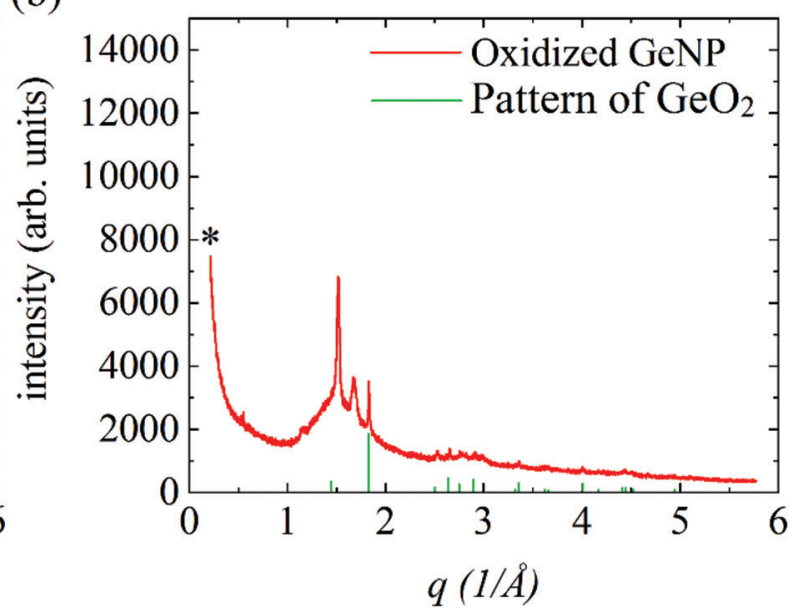

Figure 3: (a) Powder XRD pattern of germanium nanoparticles. The star marks particle-particle correlations (see text). (b) Powder XRD pattern of germanium nanoparticles denotes the reflections of $\mathrm{GeO}_{2}$ after overnight measurement and compared to the reference pattern of $\mathrm{GeO}_{2}$ (PDF \# 03-065-6772). The peaks at $\mathrm{q}=1.5$ and $\mathrm{q}=1.7$ belong to a crystalline phase of unknown composition.
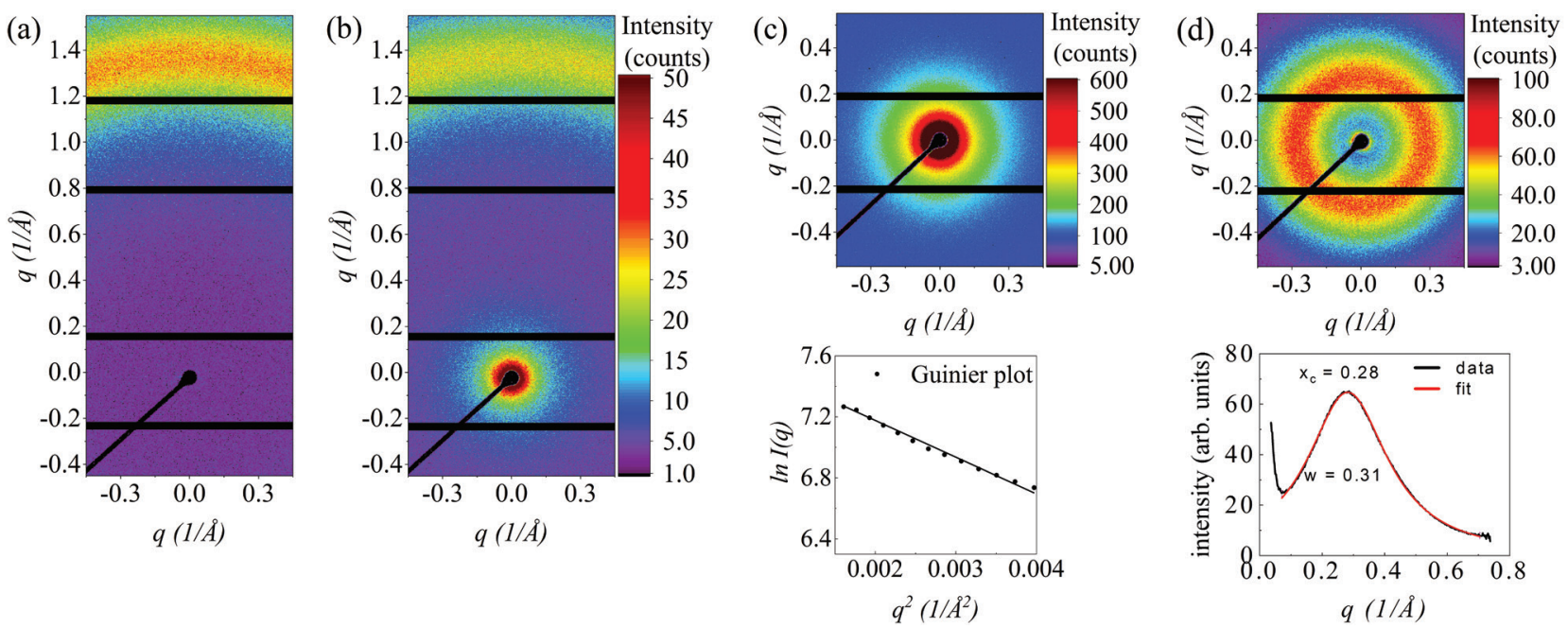

Figure 4: SAXS/WAXS reciprocal space maps of GeNP used to determine inter/intra-particle structure and distances. (a) Reciprocal map of solvent in capillary. (b) Reciprocal map of dissolved GeNP in capillary. (c, top subfigure) Reciprocal map of dissolved GeNP in capillary with longer acquisition. (c, bottom subfigure) Guinier plot obtained from reciprocal map (Figure 4c, top subfigure). (d, top subfigure) Reciprocal space map of powder GeNP in capillary. (d, bottom subfigure) Line-cut with its fit obtained by circular gathering of reciprocal space map (Figure 4d, top subfigure).

to obtain information about the size of the GeNP. More detailed information is given in Supplementary Material. The Guinier plot obtained from the measured reciprocal map is presented in Figure 4c (bottom subfigure), leading to a calculated diameter of $33.6 \AA$. SAXS measurements of the pure GeNP in powder form were carried out for comparison with SAXS measurements of dissolved GeNP in a capillary. The resulting reciprocal map is presented in Figure 4d (top subfigure).

The scattering maxima in form of a ring correspond to the average particle-particle distance and random orientation of the nanoparticles. Taking into account the random orientation of the GeNP in the powder form and nanoparticles in closest packing (NP are touching each other) it is possible to estimate the average diameter (size) of the GeNP:

$$
D=\frac{2 \pi}{x_{c}}
$$

where $x_{c}$ is the position of the measured peak in reciprocal space, i.e., in inverse Å. The corresponding circle gathered line-cut with Lorentzian peak fit and $x_{c}$ is presented in Figure $4 \mathrm{~d}$ (bottom subfigure). The position of the peak 
is in good agreement with the strongest peak measured by PXRD (Figure 3a). The calculated diameter is thereby $\mathrm{D}=44.9 \AA$. The difference between the diameter obtained by Guinier analysis and the diameter calculated from the position of the scattering maxima can be explained by relatively close packing in the powder and less densely packed GeNP dissolved in THF. Further analysis of the nanoparticles was tried via transmission electron microscopy (TEM). However, during the measurement, the GeNP decompose under the electron beam (Figure S2), indicating that the particles are not only sensitive to moisture and air but still thermally unstable. This might be due to the halide terminated surface of these particles, which is still prone to a disproportionation reaction under heat treatment. Hence, the surface termination might play an important role in the stabilization of such GeNP and further surface modifications are needed to isolate more stable particles.

\section{Conclusions}

We presented a unique and novel synthetic route for the synthesis of germanium nanoparticles (GeNP) by using the disproportionation reaction of metastable $\mathrm{Ge}(\mathrm{I}) \mathrm{Br}$ solutions. This mild method generates well-defined GeNP with a sharp size distribution in the range of 20-100 $\AA$ and with a yield of up to $79 \%$. The size of the particles can be easily controlled by using defined annealing times at certain temperatures. The resulting particles are sensitive to air and moisture. This sensitivity which can be traced back to the Ge-Br-moieties at the surface of the particles opens the door for further surfacefunctionalization. Currently, we are working on a method for such functionalization and/or stabilization of the particles for future applications of these novel germanium nanoparticles.

\section{Experimental section}

All reactions were carried out under rigorous exclusion of air and moisture using Schlenk techniques under nitrogen atmosphere. All organic solvents were dried over sodium and purified via distillation. DLS measurements were performed on a Malvern Zetasizer Nano ZS. EDX analysis was performed at a HITACHI SU8030 scanning electron microscope with Bruker-EDX using solid samples which were prepared in an Ar-filled glovebox. Elemental Analysis was performed using solid samples at a Vario
Micro cube from Elementar Analysensysteme $\mathrm{GmbH}$. Powder XRD patterns of the GeNP were measured at room temperature with a Stoe STADI-P X-ray diffractometer using monochromatized $\mathrm{Cu}-\mathrm{K}_{\alpha 1}$ radiation $(\lambda=1.540598 \AA$ 品 and a Mythen - $1 \mathrm{~K}$ detector. Standard measurements were taken in a $q$ range of $\left(0.2-6 \AA^{-1}\right)$. Powder samples were fixed under an argon atmosphere with grease between two Mylar foils.

SAXS measurements were conducted on a Xeuss 2.0 setup (Xenocs). A CuKa X-ray beam with wavelength $\lambda=1.5418 \AA(\mathrm{E}=8.04 \mathrm{keV})$ and a beam size of $\sim 500 \times 500 \mu \mathrm{m}^{2}$ (FWHM) with photon flux $10^{7} \mathrm{ph} . / \mathrm{s}$ was used. A twodimensional detector Pilatus 300K (Dectris) with $487 \times 619$ pixels of $172 \times 172 \mu \mathrm{m}^{2}$ was positioned in sample-detector distance (SDD) $367 \mathrm{~mm}$. Powder and solution samples were fixed under nitrogen atmosphere conditions.

\section{Synthesis of the particles}

The nanoparticles were prepared via disproportionation reaction of metastable subvalent $\mathrm{Ge}(\mathrm{I}) \mathrm{Br}$ solutions in the yield up to $79 \%$. Synthesis of the metastable Ge(I) $\mathrm{Br}$ solutions was done as follows: liquid germanium (40 mmol) reacted with $\mathrm{HBr}(40 \mathrm{mmol})$ at approximately $1600^{\circ} \mathrm{C}$ under high vacuum conditions (Schnepf, 2005). The resulting gaseous products condensed with $200 \mathrm{~mL}$ of a 10 to 1 mixture of $\mathrm{THF} / \mathrm{P}^{\mathrm{n}} \mathrm{Bu}_{3}$ on a surface at $-196^{\circ} \mathrm{C}$. After the solid matrix had been warmed to $-78^{\circ} \mathrm{C}$, an orange-reddish solution was obtained. This solution was slowly warmed up to room temperature under stirring. The hydrodynamic particle diameter was measured using DLS and was in a range of 20-40 A. The solution was heated for $16 \mathrm{~h}$ at $55^{\circ} \mathrm{C}$. The heating process increases the hydrodynamic diameter of germanium nanoparticles up to $100 \AA$. All volatiles were removed under vacuum to give an oily reddish substance, which was washed up to six cycles with pentane and then dried under vacuum. Afterwards, the obtained dark red powder was dissolved in THF and filtered through a filter cannula (filter paper: pore size 4.0-12.0 $\mu \mathrm{m}$, grade MN 615) to remove the agglomerations of non-dispersible particles. The dissolution of the powder in THF results in a dark red colloidal solution with the before chosen hydrodynamic particle diameter from 20 to 100 Å. For elemental analysis, EDX and powder XRD, all volatiles were removed in vacuum yielding a red powder, which is very sensitive to air and water.

Acknowledgement: We gratefully acknowledge the financial support from the Topical Graduate School „Novel 
nanoparticles: from synthesis to biological applications“ at the University of Tübingen. We thank Dr. Fajun Zhang for helpful discussions, Dr. Markus Ströbele and Thorsten Hummel for providing PXRD measurements, and Prof. Dr. Chassé and Elke Nadler for recording the EDX spectra. We further acknowledge the Alexander von Humboldt foundation and the Zeiss foundation for the financial support of Dr. M. Hodas.

Funding information: Topical Graduate School „Novel nanoparticles: from synthesis to biological applications“.

Author contributions: Viktoriia Slynchuk: writing original draft, writing - review and editing, visualization, investigation, methodology, formal analysis; Martin Hodas: writing - original draft, writing - review and editing, methodology, formal analysis; Dominik NaglavHansen: writing - review and editing; Frank Schreiber: funding acquisition, writing - review and editing; Andreas Schnepf: supervision, project administration, funding acquisition, writing - review and editing.

Conflict of interest: Authors state no conflict of interest.

\section{References}

Bernard A., Zhang K., Larson D., Tabatabaei K., Kauzlarich S.M., Solvent effects on growth, crystallinity, and surface bonding of Ge nanoparticles. Inorg. Chem., 2018, 57, 9, 5299-5306. https:// doi.org/10.1021/acs.inorgchem.8b00334

Bhattacharjee S., Rietjens I.M.C.M., Singh M.P., Atkins T.M., Purkait T.K., Xu Z., et al., Cytotoxicity of surface-functionalized silicon and germanium nanoparticles: the dominant role of surface charges. Nanoscale, 2013, 5, 4870. https://doi.org/10.1039/ C3NR34266B

Campelo J. M., Luna D., Luque R., Marinas J.M., Romero A.A., Sustainable preparation of supported metal nanoparticles and their applications in catalysis. ChemSusChem, 2009, 2, 18-45. https://doi.org/10.1002/cssc.200800227

Carolan D., Doyle H., Size and emission color tuning in the solution phase synthesis of highly luminescent germanium nanocrystals. J. Mater. Chem. C, 2014, 2, 3562. https://doi.org/10.1039/ C4TC00319E

Chiu Y.-W., Huang M.H., Formation of Hexabranched $\mathrm{GeO}_{2}$ Nanoparticles via a Reverse Micelle System. J. Phys. Chem. C, 2009, 15, 6056-6060. https://doi.org/10.1021/jp9002615

Fan J., Chu P.K., Group IV nanoparticles: synthesis, properties, and biological applications. Small, 2010, 6, 2080-2098. https://doi. org/10.1002/smll.201000543

Freund R., Lächelt U., Gruber T., Rühle B., Wuttke S., Multifunctional efficiency: extending the concept of atom economy to functional nanomaterials. ACS Nano, 2018, 12, 2094-2105. https://doi. org/10.1021/acsnano.8b00932
Gerung H., Bunge S.D., Boyle T.J., Brinker C.J., Han S.M., Anhydrous solution synthesis of germanium nanocrystals from the germanium (II) precursor Ge[N(SiMe $\left.)_{2}\right]_{2}$. Chem. Commun., 2005, 1914-1916. https://doi.org/10.1039/B416066E

Goesmann H., Feldmann C., Nanoparticulate functional materials. Angew. Chem. Int. Ed., 2010, 49, 1362-1395. https://doi. org/10.1002/anie.200903053

Grzelczak M., Liz-Marzán L.M., Klajn R., Stimuli-responsive self-assembly of nanoparticles. Chem. Soc. Rev., 2019, 48, 1342-1361. https://doi.org/10.1039/C8CS00787)

Johnson G.M., Weller M.T., A powder neutron diffraction study of lithium-substituted gallosilicate and aluminogermanate halide sodalities. Inorg. Chem., 1999, 38, 2442-2450. https://doi. org/10.1021/ic9812510

Lambert T.N., Andrews N.L., Gerung H., Boyle T.J., Oliver J.M., Wilson B.S., et al., Water-Soluble Germanium (0) Nanocrystals: Cell Recognition and Near-Infrared Photothermal Conversion Properties. Small, 2007, 3, 691-699. https://doi.org/10.1002/ smll.200600529

Lin W., Introduction: nanoparticles in medicine. Chem. Rev., 2015, 115, 10407-10409. https://doi.org/10.1021/acs.chemrev.5b00534

Loza K., Heggen M., Epple M., Synthesis, structure, properties, and applications of bimetallic nanoparticles of noble metals. Adv. Funct. Mater., 2020, 30, 1909260. https://doi.org/10.1002/ adfm.201909260

McVey B.F.P., Prabakar S., Gooding J.J., Tilley R.D., Solution synthesis, surface passivation, optical properties, biomedical applications, and cytotoxicity of silicon and germanium nanocrystals. ChemPlusChem, 2017, 82, 60. https://doi. org/10.1002/cplu.201600207

Modena M.M., Rühle B., Burg T.P., Wuttke S., Nanoparticle Characterization: Nanoparticle Characterization: What to Measure? Adv. Mater., 2019, 31, 1901556. https://doi.org/10.1002/ adma.201970226

Rogach A., Eychmüller A., Hickey S., Kershaw S., Infrared-emitting colloidal nanocrystals: synthesis, assembly, spectroscopy, and applications. Small, 2007, 3, 536-557. https://doi.org/10.1002/ smll.200600625

Ruddy D.A., Johnson J.C., Smith E.R., Neale N.R., Size and bandgap control in the solution-phase synthesis of near-infrared-emitting germanium nanocrystals. ACS Nano, 2010, 4, 7459-7466. https://doi.org/10.1021/nn102728u

Scheele M., Brütting W., Schreiber F., Coupled organic-inorganic nanostructures (COIN). Phys. Chem. Chem. Phys., 2015, 17, 97-111. https://doi.org/10.1039/C4CP03094J

Schnepf A., Köppe R., Synthesis of Germanium (I) Bromide. A First Step Towards New Germanium Cluster Compounds? Z. Anorg. Allg. Chem., 2002, 628, 2914-2918. https:// doi.org/10.1002/1521-3749(200213)628:13<2914::AIDZAAC2914>3.0.CO;2-0

Schnepf A., On the Redox Chemistry of Gel Bromide. Eur. J. Inorg. Chem., 2005, 2120-2123. https://doi.org/10.1002/ ejic. 200400843

Shirahata N., Hirakawa D., Masuda Y., Sakka Y., Size-dependent color tuning of efficiently luminescent germanium nanoparticles. Langmuir, 2013, 29, 7401-7410. https://doi.org/10.1021/ la303482s

Smock S.R., Tabatabaei K., Williams T.J., Kauzlarich S.M., Brutchey R.L., Surface coordination chemistry of germanium nanocrystals 
synthesized by microwave-assisted reduction in oleylamine. Nanoscale, 2020, 12, 2764-2772. https://doi.org/10.1039/ C9NR09233A

Sodreau A., Lentz N., Frutos M., Mallet-Ladeira S., Nayral C., Delpech F., et al., Symmetric and non-symmetric bis-metallylene iron complexes, precursors of iron germanide nanoparticles. Chem. Commun., 2019, 55, 9539-9542. https://doi.org/10.1039/ C9CC05063A

Stratakis M., Garcia H., Catalysis by supported gold nanoparticles: beyond aerobic oxidative processes. Chem. Rev., 2012, 112, 4469-4506. https://doi.org/10.1021/cr3000785

Talapin D.V., Lee J.S., Kovalenko M.V., Shevchenko E.V., Prospects of colloidal nanocrystals for electronic and optoelectronic applications. Chem. Rev., 2010, 110, 389-458. https://doi. org/10.1021/cr900137k
Uno R., Ozawa H., Yamanaka T., Morikawa H., Ando M., Ohsumi K., et al., Powder Diffractometry at the Tsukuba Photon Factory. Aust. J. Phys., 1988, 41, 133-144. https://doi.org/10.1071/PH880133

Wang Y., Ramesh U., Nyamekye C.K.A., Ryan B.J., Nelson R.D., Alebri A.M., et al., Synthesis of germanium nanocrystals from solid-state disproportionation of a chloride-derived germania glass. Chem. Commun., 2019, 55, 6102-6105. https://doi. org/10.1039/C9CC01676G

Weller H., Quantized semiconductor particles: a novel state of matter for materials science. Adv. Mater., 1993, 5, 88-95. https://doi. org/10.1002/adma.19930050204

Wu S., Han C., locozzia J., Lu M., Ge R., Xu R., et al., Germaniumbased nanomaterials for rechargeable batteries. Angew. Chem. Int. Ed., 2016, 55, 7898-7922. https://doi.org/10.1002/ anie. 201509651 